\title{
Analysis of micro vibration in gas film of aerostatic guide way based on molecule collision theory
}

\author{
Shao Hua YANG ${ }^{1}$, Wei LONG ${ }^{1, a}$ and Ling GONG ${ }^{1}$ \\ ${ }^{1}$ Faculty of mechanical and electrical engineering, Kunming University of Science and Technology, Yunnan Kunming 650500, China
}

\begin{abstract}
Micro vibration of the aerostatic guide way has a significant impact on its dynamic characteristics and stability, which limits the development of pneumatic component. High pressure gas molecules have been collided with the supporting surface and the internal surface of the throttle during the flow process. Variable impulse of the surfaces aside for the gas film are affected by the changes of impulse which formed irregular impact force in horizontal and vertical direction. Micro-vibration takes place based on the natural frequency of the system and its frequency doubling. In this paper, the vibration model was established to describe the dynamic characteristics of the gas film, and the formation mechanism of micro vibration in the film is defined. Through the simulation analysis and experimental comparison, formation mechanism of the micro vibration in the gas film is confirmed. It was proposed that the micro vibration of gas film can be produced no matter whether there is a gas chamber or not in the throttle. Under the same conditions, the micro vibration of the guide way with air chamber is greater than that without any chamber. The frequency points of the vibration peaks are almost the same, as well as the vibration pattern in the frequency domain.
\end{abstract}

\section{O INTRODUCTION}

With the development of ultra precision motion platform, micro vibration has become a major problem which restricts the development of air flotation technology. Studying the mechanism of micro vibration is very important for improving the movement and positioning accuracy of air floatation platform[1-3]. Micro vibration is different from the "air hammer phenomenon" from the static pressure guide, It is mainly due to the gas film clearance between the film and the internal support platform of uneven force distribution has certain amplitude near the equilibrium position of the law on the deflection or vibration. Aoyamae et al. It is speculated that the micro vibration in gas bearing is caused by the flow of gas in the pressure chamber[4]: Chenxuedong et al. Through the simulation experiments, it is proved that the phenomenon of the air cavity of the small orifice throttle air pressure guide rail is related to the micro vibration, Gas flow in the gas film can also cause the micro vibration, but less than the micro vibration of the cyclone[5]; Zhang Ming et al. Think that the main reason of the micro vibration in the ultra precision working table system of the air bearing is that the turbulence of the gas flow in the gas flow is formed and the measures to restrain the vibration of the micro vibration [6].Dong Ju Chen et al by Reynolds equation derived row orifice form hydrostatic slideway of the gas film stiffness formula and Study on machining accuracy of workpiece caused by fluctuation of gas membrane effect[7].Ji Lujun et al. Use the linear perturbation method to establish the stability of the Reynolds equation and the Reynolds equation and the bearing motion equation to study the dynamic analysis model of the vertical impact caused by the static pressure rail fluctuations[8]. The amplitude of the micro vibration is typically between a few nanometers to tens of nanometers, and the frequency range from tens of hertz to several thousand hertz. For the cause of the vibration of the micro vibration, it is generally accepted that the gas flow pattern of the gas is caused by the flow of the gas, But there is no reasonable explanation for this kind of fluctuation, In this paper, the point of local impulse is changed from the velocity vector of gas molecule collision, and the micro vibration of the gas film is described by the simulation and experiment, This paper provides a new way of thinking for the fundamental suppression of gas film fluctuation.

\section{Study on formation mechanism of gas film fluctuation}

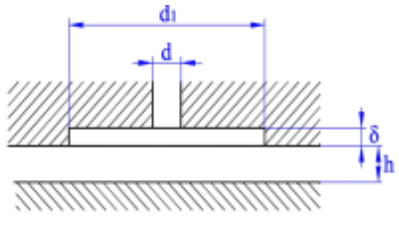

(a) orifice throttling 


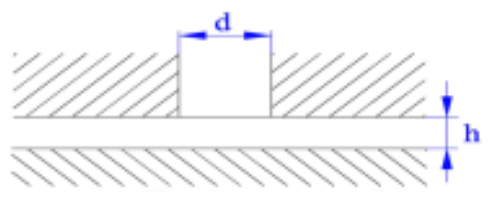

(b) torus throttling

Figure 1. common throttle.

Figure 1 (a) is shown as a small orifice throttling view, figure 1 (b) is a torus throttling view, In this paper, the characteristics of the gas flow in a complex throttle device with a gas chamber are described, The torus throttle represented that no flow law of gas cavity internal gas throttling device

The orifice restrictor, external high-pressure gas into the gas chamber through the gas supply hole, gas velocity vector does not change in the high pressure jet, between the molecules by diffusion, the colliding molecules directly into the gas chamber, high pressure jet impact on the cavity at the bottom of the gas molecules From the beginning of the impact hypothesis, all the kinetic energy into pressure energy, The speed of the molecules that impact the bottom of the gas chamber is reduced, and the bottom temperature can be increased, which makes this part of the surface heat flow occurs at the instantaneous maximum, The support at the bottom of the thermal strain and cause fluctuation of gas film. The molecular impact area is $\mathrm{A}$, then there is:

$$
\frac{\rho A l v^{2}}{2}=\frac{A l \Delta p_{\text {max }}^{2}}{2 E}
$$

In this formula: 1 is the equivalent gas film thickness of the gas chamber, $l=h+\delta ; \mathrm{E}$ is a gas volume elastic modulus; $c$ is the local speed of sound, $c=\sqrt{E l \rho} ; \Delta p_{\max }$ is the peak value of pressure; $\Delta p_{\max }=p v \sqrt{E / \rho}=\rho c v$, The energy absorbed by the elastic deformation is not considered in the derivation process, and the actual $\Delta p_{\max }$ is smaller than the calculated value.

The interaction between the gas molecules and the wall of the supporting surface is affected, Supposed that the $\sigma_{v}$ part of the stream is completely diffuse, Then the rest of the $\left(1-\sigma_{v}\right)$ part is completely specular reflection. Specular reflection and diffuse reflection to the direction of movement of gas moleculars change. It is assumed that the molecules of the surface of the left surface are scattered by the Maxwell distribution, and the velocity distribution function is:

$$
\begin{aligned}
& f_{0}=n\left(\frac{m}{2 \pi k T}\right)^{3 / 2} \exp \left(-\frac{m}{2 k T} c^{\prime 2}\right) \\
& =n\left(\frac{m}{2 \pi k T}\right)^{3 / 2} \exp \left[-\frac{m}{2 \pi k T}\left(u^{\prime 2}+v^{\prime 2}+\omega^{\prime 2}\right)\right]
\end{aligned}
$$

In this formula: $n$ is the molecular number density, $m$ is molecular weight, $c$ is the molecular heat movement speed, $k$ is Boltzmann constant, $T$ is the thermodynamic temperature in equilibrium state.

Since the $\mathrm{H}$ is micron level, Small part of the molecule after collision between molecules and the clearance of gas film inner surface collision fold, And then directly into the gas film thickness of $\mathrm{h}$; Most molecules collide with each other, and the gas molecules collide with the surface of the gas, The velocity vector of the gas chamber is changed dramatically, and the impulse of the wall is changed rapidly, so that the wall is not balanced.

For torus throttling, The law of the movement of the gas molecules is similar to that of the molecular motion of the orifice, But because of the small hole in the wall, the pressure in the horizontal, vertical, and the velocity of the molecules is increased, and the pressure of the air floating block is not balanced in the three-dimensional direction. The main source of the micro vibration of the torus is the energy conversion of the high pressure jet molecules and the energy conversion and the imbalance of the gas molecules in the vertical direction after the impact of the support surface. The mechanism of the micro vibration of the static pressure guide rail is analyzed from the gas flow pattern, which is caused by the impact of the high pressure gas and the wall of the throttle.

The molecular interaction between molecules and the surface of the film space in the collapse of the collision, After entering the real meaning of the supporting lubrication region, the gas molecules in the pressure difference, the velocity vector in the horizontal component is much larger than the vertical direction, The change of molecular velocity vector is small, and the degree of micro vibration caused by this is very weak.

\section{Simulation Analysis and experimental verification}

\section{1 simulation analysis}

In this paper, the geometric parameters are used to enter the hole diameter $d=0.15 \mathrm{~mm}$ and the gas chamber diameter is $d=1 \mathrm{~mm}$, Torus throttle orifice floating block gas cavity height $\delta=0.1 \mathrm{~mm}$ floatation block and inlet diameter of $d=0.15 \mathrm{~mm}$, In the gas supply pressure of $0.3 \mathrm{MPa}$ and $0.6 \mathrm{MPa}$, the air gap is $30 \mu \mathrm{m}$ The results of CFD software simulation as shown in figure 2, With the increase of gas supply pressure, the inlet gas velocity of the throttle orifice and the torus is increased, the momentum of the vertical or the surface of the support is changed dramatically, which is characterized by the increase of the gas pressure. By the figure 2 (d) (c), the velocity vector is changed, the cyclone is formed in the gas chamber, with the increase of inlet pressure, the molecular velocity vector of the cyclone and the change of the surface impulse in the gas chamber. By (a) (c), (b) (d), it can be known that the presence of the gas chamber makes the molecular velocity of the gas chamber is larger than that of the same range, and the speed vector changes dramatically. Fig. 3 shows that the velocity of the gas molecules is greater than that of the gas flow in the center of the high pressure gas inlet. Because the simulation of CFD software is based on the assumption that the 
simulation results of the laminar turbulence model are obtained, it is not be very accurate to simulate the motion trajectory of molecules with the collision avoidance, Figure 4 is the use of LAMMPS software to simulate the flow of molecules with the wall surface after the collision, the molecular velocity vector changes. It can be seen that the velocity vector of the molecules of the high speed movement changes sharply, resulting in the rapid change of the wall, which makes the stress of the wall is not balanced.

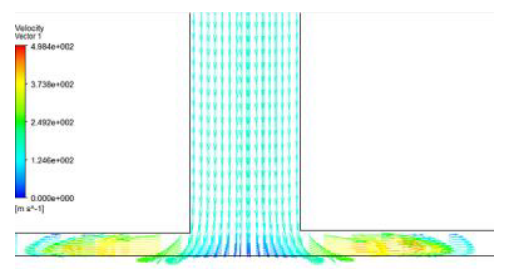

(a) $\mathrm{P}=0.3 \mathrm{MPa}$ torus throttling speed vector variation

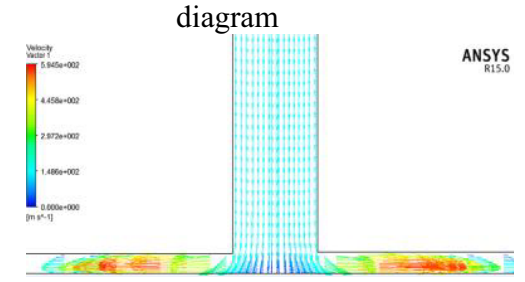

(b) $\mathrm{P}=0.6 \mathrm{MPa}$ torus throttling speed vector variation diagram

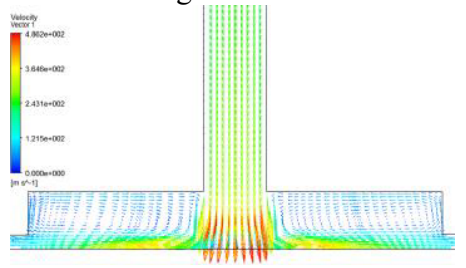

(c) $\mathrm{P}=0.3 \mathrm{MPa}$ orifice throttling velocity vector variation

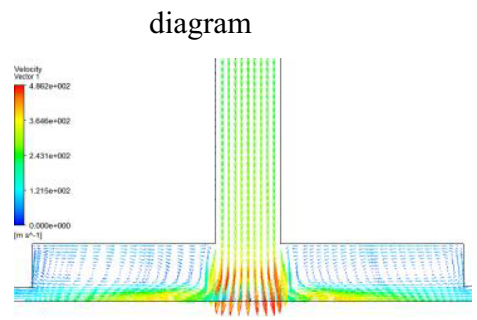

(d) $\mathrm{P}=0.6 \mathrm{MPa}$ orifice throttling velocity vector variation diagram

Fig 2. the variation of the internal velocity vector of two kinds of throttle under different inlet pressure.

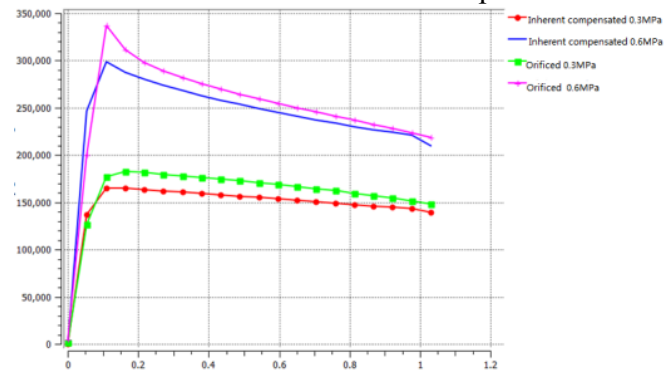

Fig 3 . gas molecular velocity diagram on the axis of the inlet center.

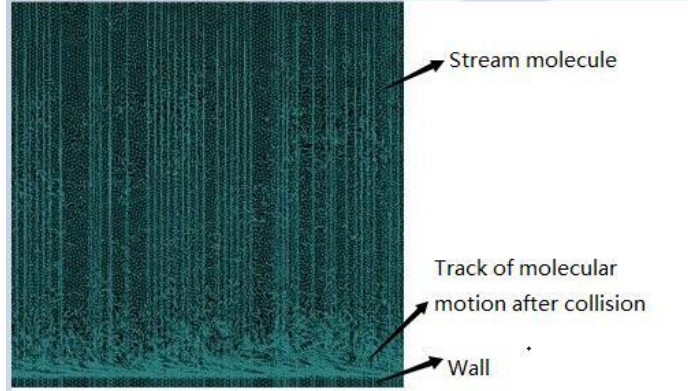

Fig 4. LAMMPS simulation to flow molecular and wall collision trajectories.

\subsection{Experimental verification}

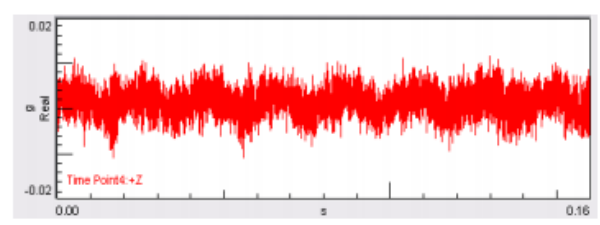

(a) $\mathrm{P}=0.3 \mathrm{Mpa}$ small hole throttle floating support signal

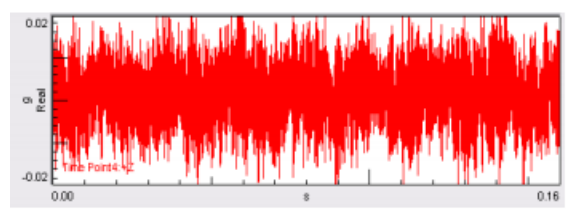

(b) $\mathrm{P}=0.6 \mathrm{Mpa}$ small hole throttle floating support signal

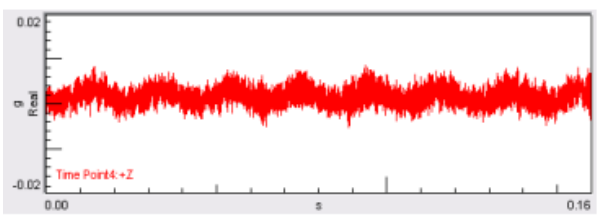

(c) $\mathrm{P}=0.3 \mathrm{Mpa}$ torus throttle floating support signal

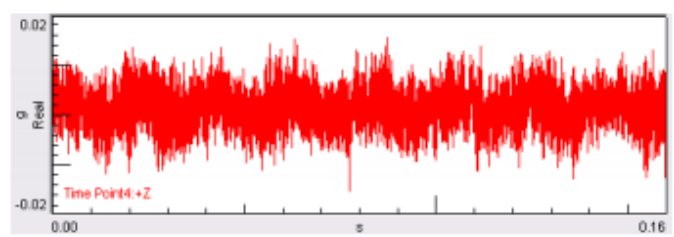

(d) $\mathrm{P}=0.6 \mathrm{Mpa}$ torus throttle floating support signal

Figure 5 .orifice and torus throttle floating support signal. Figure 5 (a) (c) respectively as the gas supply pressure is $0.3 \mathrm{MPa}$, gas film clearance $\mathrm{h}$ is about 30 , the orifice and torus throttle floating support time domain signal. Figure 5 (b) (d) respectively as the gas supply pressure is $0.6 \mathrm{MPa}$, gas film clearance $\mathrm{h}$ is about 50 , orifice and torus throttle floating support time domain signal. Under the same air supply pressure and gas film clearance did not differ much, degree of micro vibration of throttle orifice is about 2 times the torus throttle micro vibrational degrees of, with the increase of the air pressure, small hole throttle cavity and the torus knots flow increased speed of gas molecules within the scope of the hole at the end of, fierce collision and velocity changes more frequently, rail surface by pressure variation has become even more unbalanced, the degree of micro vibration becomes large. 
Figure 6 is a diagram of the orifice and the frequency domain signal torus throttle floating vibration of support, which is similar to the vibration between the two laws, The peak signal of vibration is $16 \mathrm{KHZ}, 25 \mathrm{KHZ}, 32 \mathrm{KHZ}$, $38 \mathrm{KHZ}, 45 \mathrm{KHZ}$, these frequencies are close to the natural frequency of air flotation system. In the low frequency range, the peak phase difference between the orifice and the torus is larger than that of the high frequency section. From the experimental results, we can know that there exists a micro vibration phenomenon in the throttle and the torus, but because of the existence of the hole wall, the velocity vector of the three-dimensional direction of the hole is increased, which makes the micro vibration of the orifice is greater than that of the torus.

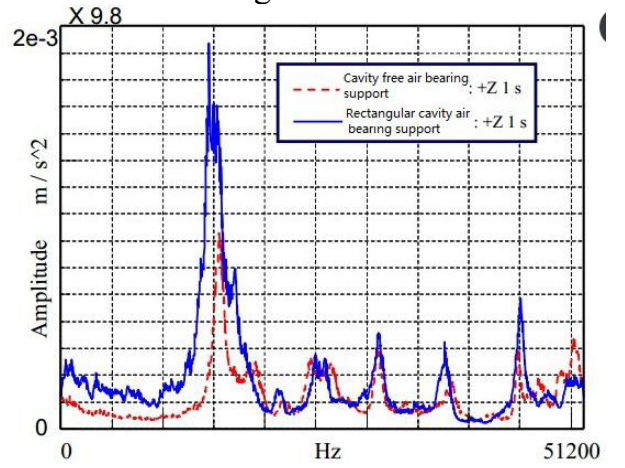

Figure 6. orifice and torus throttle floating support frequency signal.

\section{Conclusions}

(1) In this paper, the mechanism of micro vibration of static pressure guide rail is analyzed from the gas flow pattern, because of the impact of the high pressure gas and the wall and the supporting bottom surface of the throttle, the change of the velocity vector in the vicinity of the throttle is different, and the variation of the vector is different, which makes the interaction between the air floating block and the supporting surface is not balanced, resulting in the phenomenon of micro vibration.

(2) The micro vibration is generated by the air flotation support of the throttle orifice and the torus throttling, just because of the existence of gas cavity, it exacerbated by the complexity of the film inside the solid wall structure, and the influence of the molecular motion speed of air flow and change more, Performance of macro internal air cavity air film vibration is gas cavity is more obvious. The study also shows that although the micro vibration of the gas chamber is greater than that of the non gas cavity, but the same gas supply and load conditions, the vibration peak of the frequency points are basically the same; At low frequencies, there is a lot of vibration peak (nearly 2 times) of the throttle with a gas chamber.

(3) With the gas pressure increasing, the molecular velocity of the incident gas increases. The molecular and the support surface or the surface of the gas chamber, and the gas film internal gas molecular velocity vector changes dramatically, The component perpendicular to the support table or gas cavity surface changes rapidly, and the film inside the micro vibration severity.

\section{Thanks}

We show great acknowledgement of the experimental data from degree thesis of Dr. Ye Yixi" Study on ultra precision motion platform in support of the vibration characteristics of the floating ".

\section{References}

1. Zhu Yu, Wen,Sheng, Duan Guanhong . Research on ultra-Precision Stages of lithography. Lithography technology [J].2004.33(2).

2. Shinno H, Yoshioka H, Taniguchi K.A newly developed linear motor-driven aerostatic X-Y planar motion table system for nano-manchining. Annals of the CIRP,2007,56(1)

3. Verma Shobhit, Kim Won-Jong, Shakir Huzefa .Mu lti-axis maglev nanopositioner for precision manufac turing and manipulation applications. IEEE Transacti ons on Industry Applications,2005,41(5).

4. Aoyama T, Kakinuma Y, Kobayashi Y. Numerical and experimental analysis for the small vibration of aerostatic guideways. CIRP Annals-Manufacturing Technology[J], 2006,55(1).

5. [5] Chen Xuedong, He Xueming . The effect of the recess shape on performance analysis of the lubricated bearing in optical lithography, Tribology International[J], 2006,39(11).

6. Zhang Ming, Zhu Yu, Duan Guanhong . Microvibration of Ultra-precision Gas Bearing Linear Motion Stage and Its Elimination. Design and research [J].2005, (11).

7. Chen Dongju, Zhang Feihu , Fu Pengqiang . Identific ation Fluctuation of Aerostatic Guideway. Journal of Mecheanical Engineering[J].2010,46(21).

8. Ji Lujun, Zhang Ming, Hu Jinchun, Zhu Yu. Stabilit y to vertical impulse perturbation of plane externally pressrize gas bearings. Tsing Univ(Sci\&Tech)[J].201 $1,51(6)$. 